\title{
Patrones de riqueza altitudinal de Papilionidae, Pieridae y Nymphalidae (Lepidoptera: Rhopalocera) en áreas montañosas de México
}

\author{
David Monteagudo Sabaté \& Moisés Armando Luis Martínez* \\ Museo de Zoología, Departamento de Biología Evolutiva, Facultad de Ciencias, Universidad Nacional Autónoma de \\ México, Apartado Postal 70-399, 04510, México, D.F. México; dmonteagudo@ciencias.unam.mx, \\ alm@ciencias.unam.mx \\ * Correspondencia
}

Recibido 20-VIII-2012. Corregido 08-I-2013. Aceptado 08-II-2013.

\begin{abstract}
Altitudinal richness patterns of Papilionidae, Pieridae and Nymphalidae (Lepidoptera) in Mexican mountain areas. Butterflies constitute an useful group to investigate biodiversity patterns in specific geographic areas. The aim of this study was to describe the altitudinal patterns distribution and to recognize the main grouping factors of these families. We conducted a comparative study between the butterfly fauna (Papilionidae, Pieridae and Nymphalidae) of five Mexican mountain ranges (Sierra de Manantlán, Sierra de Atoyac de Álvarez, Loxicha Region, Teocelo-Xalapa and Sierra de Juárez), that included 34 sites of altitudinal ranges from 100 to $2820 \mathrm{~m}$. Data was obtained from the Zoology Museum of the National University of Mexico, and comprised more than 60000 butterfly records of 398 taxa (subspecies level) proceeding during the last 35 years. Fauna similarity between localities were analyzed using a cluster analysis by Sorensen similarity coefficient. Species richness showed a general tendency to decrease with altitude; the main difference was found between the locality with higher altitude and the rest of the sites. The principal factors affecting the identified clusters followed this order: the location in Pacific or Atlantic slope, and location on a particular mountain range. Three altitudinal levels (low elevations, up to $1200 \mathrm{~m}$; intermediate elevations, from 1200 to $1800 \mathrm{~m}$; and high elevations, from 1800 to $2500 \mathrm{~m}$ ) were described in accordance to their main characteristic taxa. While Neartic elements were common in the highest altitudinal floor, Neotropical taxa were common in the lowest one. It was more difficult to characterize the intermediate level in which a high number of localities were clustered; this intermediate level was characterized by the presence of some endemic species. The results suggest that historical factors are preeminent in butterfly fauna composition in these areas. Future studies may include other Mexican mountain areas to obtain more information on the different factors (latitude, altitude, slope) influencing biodiversity patterns. Rev. Biol. Trop. 61 (3): 1509-1520. Epub 2013 September 01.
\end{abstract}

Key words: Papilionoidea, butterflies, altitudinal distribution, faunistic similarity, Sørensen similarity coefficient.

Las mariposas diurnas integran uno de los grupos más utilizados para el reconocimiento y monitoreo de la diversidad de una biota, esto es debido fundamentalmente a que es un grupo del que se posee un buen conocimiento sistemático, es conspicuo, abundante, de fácil recolección y determinación taxonómica en el campo (Llorente et al. 1994). Las listas locales o regionales de faunas de mariposas son un objeto excelente para emplearse en trabajos de índole ecológica y biogeográfica (Luis et al. 2005).

Desde 1974, el Museo de Zoología de la Facultad de Ciencias (MZFC) de la Universidad Nacional Autónoma de México (UNAM) está llevando a cabo el proyecto "Estudio de las Faunas de Islas Montañosas Húmedas de México", cuyo principal objetivo ha sido la caracterización faunística del bosque mesófilo de montaña (BMM) (Rzedowski 1978), también 
reconocido como bosque nuboso (CONABIO 2010). Resultado de este proyecto, se han publicado listas lepidóptero-faunísticas provenientes de transectos altitudinales que van de los 600 a los $3100 \mathrm{~m}$ de altitud y de vegetación en las siguientes zonas: Área Montañosa de Teocelo-Xalapa (Llorente et al. 1986), Sierra de Juárez (Luis et al. 1991), Sierra de Atoyac de Álvarez (Vargas et al. 1992) y Sierra de Manantlán (Vargas et al. 1999), las cuales se utilizan en el presente estudio comparativo. Además, se incluyeron los datos inéditos procedentes del trabajo de campo realizado en la región de Loxicha (2005-2011) y los registros de la base de datos MARIPOSA (Luis et al. 2005), con la finalidad de complementar los inventarios.

Con anterioridad se han efectuado comparaciones de similitud faunística entre distintas áreas con base en lepidóptero-faunas (Balcázar 1993, Monteagudo et al. 2001), que corroboran a grandes rasgos los patrones biogeográficos señalados por algunos autores para México (Hoffmann 1933, Beutelspacher 1983, Llorente 1984, Halffter 1987). Algunos de los factores determinantes de la distribución de estas faunas son: la compleja orografía de la Zona de Transición Mexicana (Halffter 1976, Halffter 1987), donde se entremezclan elementos neárticos, neotropicales y mesoamericanos, cuya dominancia varía de acuerdo con el gradiente latitudinal-altitudinal; el efecto de montaña, que es más marcado en áreas aisladas y que se manifiesta principalmente en los pisos de vegetación, los cuales comprenden un menor intervalo altitudinal y por último sus características históricas, como es la diferenciación de las faunas de la vertiente pacífica y atlántica (de acuerdo con las ideas de Llorente 1984 y Balcázar 1993).

El objetivo de la presente contribución es describir la relación de la riqueza del taxon con la altitud, detallar las relaciones existentes entre los inventarios procedentes de transectos altitudinales en las cinco unidades geográficas de estudio, y definir a partir de estas comparaciones y algunos táxones característicos los pisos altitudinales para las lepidóptero-faunas.

\section{MATERIALES Y MÉTODOS}

Área de estudio: Se estudiaron cinco transectos altitudinales en áreas montañosas en ambas vertientes en México; en la vertiente pacífica: la sierra de Manantlán (Colima y Jalisco), la sierra de Atoyac de Álvarez (Guerrero) y la región de Candelaria-Loxicha (Oaxaca), pertenecientes a la Sierra Madre del Sur; en la vertiente atlántica: la zona montañosa de Teocelo-Xalapa (Veracruz) y la Sierra de Juárez (Oaxaca) (Fig. 1). Estas áreas se

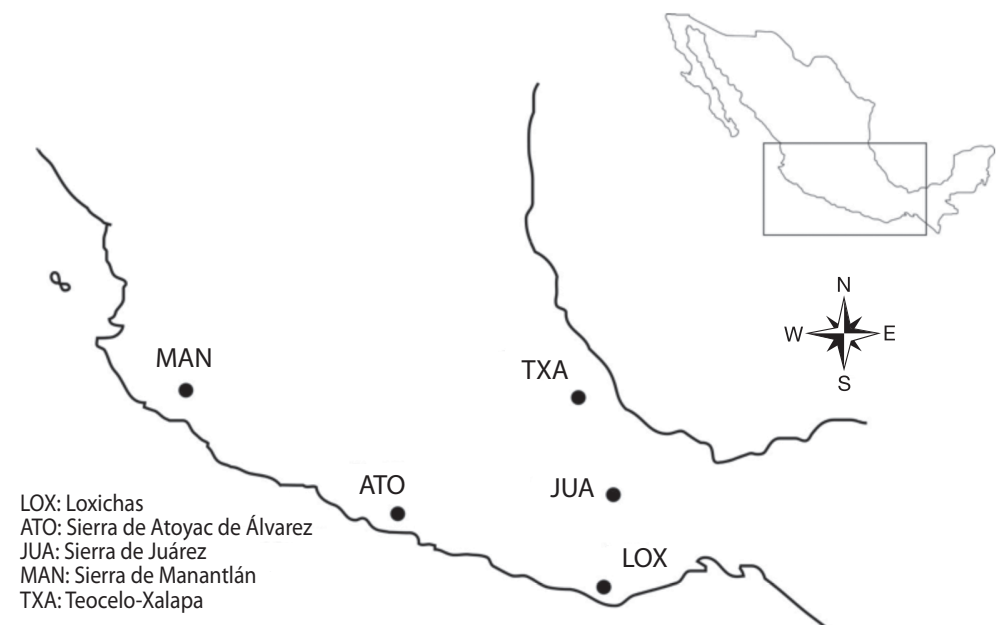

Fig. 1. Ubicación geográfica relativa de las áreas evaluadas.

Fig. 1. Relative geographical location of the mountain ranges evaluated. 
caracterizan por tener un elevado grado de humedad, lo que favorece la presencia en algunas localidades del BMM, tipo de vegetación muy variable atendiendo a sus componentes (Rzedowski 1978), de distribución fragmentada y relictual en México (Llorente 1984, Luna et al. 1989, Luna \& Alcántara 2001) y de sumo interés en cuestiones de conservación (CONABIO 2010) por su gran número de endemismos.

Los transectos se ubicaron en diferentes sistemas montañosos. Las estaciones de muestreo se establecieron en función a los diferentes pisos altitudinales, la composición de la estructura de la vegetación, a los gradientes climáticos (temperatura-humedad) y en las áreas con mayor representación de táxones endémicos de acuerdo con su altitud y tipo de vegetación.

El cuadro 1 muestra la descripción de las unidades fisiográficas estudiadas. En el cuadro 2 se presenta una breve descripción de las localidades utilizadas en el presente estudio para todos los transectos analizados.

Tratamiento de los datos: Para la realización de este estudio, se escogieron cinco transectos, con la finalidad de efectuar un estudio sobre los patrones de riqueza altitudinal de la fauna de mariposas diurnas de Papilionidae, Pieridae y Nymphalidae (Papilionoidea). Primero se requirió que cada uno de los transectos tuviera condiciones similares en cuanto al trabajo de campo realizado en cada uno de ellos, en función al esfuerzo de recolecta realizado por localidad (número de horas-hombre que fueron empleadas). De esta forma, en cuatro de los cinco transectos, se realizaron curvas de acumulación de especies para establecer el porcentaje del total que se obtuvo (Vargas et al. 1992, Soberón \& Llorente 1993, Vargas et al. 1999, Pozo et al. 2003) y poder establecer una comparación entre ellas. Cada uno de los transectos se caracteriza porque:

- Proceden de trabajos lepidóptero-faunísticos que ha realizado el personal del Museo de Zoología, en los diferentes transectos. Ello implica un método sistemático en el trabajo de campo y en el tratamiento y manejo de los datos, aparte de una seguridad en la determinación y catalogación de los ejemplares.

- Los trabajos de campo, han sido realizados en los últimos 35 años, en su mayoría con la misma metodología, con la participación de cuatro a cinco personas por transecto, dos de las cuales siempre intervinieron en cada uno de los estudios.

- Todos los casos corresponden a transectos altitudinales, con cambios en la estructura de la vegetación conforme el ascenso altitudinal, aun cuando éstos no implican el mismo intervalo altitudinal. Cada estudio comprende las distintas épocas del año a lo largo de varios años, lo cual permite extraer el factor relativo a la estacionalidad de las recolectas y fenología de la fauna.

- Cada una de las listas, se han obtenido con métodos similares de muestreo (uso de la red aérea combinado con trampas Van Someren-Rydon, un promedio de 15 por día).

- Todos los ejemplares, se encuentran depositados y catalogados en el Museo de Zoología y los datos de cada ejemplar están registrados en la base de datos MARIPOSA (aproximadamente 60000 registros entre los cinco estudios).

- Para el presente trabajo se consideran sólo los inventarios obtenidos por localidad a partir de un esfuerzo de muestra superior a 15 días de colecta por localidad -con excepción de los inventarios procedentes de Juárez Alto-. Cuando ha sido necesario, se han reunido en una sola localidad los resultados de estaciones de muestreo que, por sus características de altitud, tipo de vegetación y distancia, lo permitían. El tamaño regular de las localidades, que en este caso establecemos con respecto al esfuerzo de muestra, es necesario para que sean comparables en un estudio conjunto tal y como han señalado Murguía \& Rojas (2001).

El trabajo comparativo entre regiones y localidades se desarrolló con las familias 
CUADRO 1

Descripción de las unidades orográficas comparadas

TABLE 1

Description of the mountain ranges that are compared

Sierra de Manantlán (MAN), Colima y Jalisco.

Ubicación: entre los $19^{\circ} 26^{\prime}-19^{\circ} 42^{\prime}$ N-103 $51^{\circ}-104^{\circ} 27^{\prime} \mathrm{W}$.

Topografía: accidentada, con una altitud de $2860 \mathrm{msnm}$

Suelos: tipo regosol (entisol), cambisol (inceptisol) y litosol.

Clima: Tres subtipos: cálido subhúmedo, semicálido subhúmedo y templado subhúmedo; vertiente orientada al mar mucho más húmeda. Lluvias de junio a octubre, con una precipitación anual entre 575 y 1700mm, según localidades.

Vegetación: bosque mesófilo de montaña, bosque tropical caducifolio y bosque tropical subcaducifolio.

Trabajo de campo: sistemático en seis estaciones de muestreo, entre abril de 1989 y marzo de 1996, con 200 especies.

Sierra de Atoyac de Álvarez (ATO), Guerrero.

Ubicación: entre los $17^{\circ} 15^{\prime}-17^{\circ} 45^{\prime} \mathrm{N}-100^{\circ} 10^{\prime}-100^{\circ} 20^{\prime} \mathrm{W}$.

Topografía: muy accidentada, la mayor elevación es Cerro Teotepec (3550 msnm).

Suelos: cambisol, litosol, feozem, andosol y acrisol.

Clima: fuertemente determinado por las formas del relieve y la altitud; precipitación media anual entre 1000 y $2000 \mathrm{~mm}$, con mayor humedad en la región orientada al Pacífico que en la más continental. Los tipos de clima que se identifican en la sierra son Aw cálido subhúmedo con lluvias en verano, $\mathrm{Aw}_{2}(\mathrm{w})$ con mayor grado de humedad, $(\mathrm{A}) \mathrm{C}(\mathrm{m})(\mathrm{w})$ semicálido húmedo con lluvias abundantes en verano, $\mathrm{Cm}$ templado húmedo y $\mathrm{Cw}$ templado subhúmedo.

Vegetación: bosque tropical subcaducifolio, bosque mesófilo de montaña, bosque de encino-bosque de lauráceas y bosque de pino y encino, con distinto grado de perturbación por las presencia de cafetales en las zonas bajas.

Trabajo de campo: entre mayo de 1982 y mayo de 1985, y se distribuyen en nueve localidades, con 208 especies.

Región de los Loxichas (LOX), Oaxaca.

Ubicación: entre los $15^{\circ} 31^{\prime}-16^{\circ} 03^{\prime} \mathrm{N}-96^{\circ} 25^{\prime}-96^{\circ} 31^{\prime} \mathrm{W}$.

Topografía: paisaje típico de macropendiente de las montañas, formada por la elevación tectónica del terreno en el mioceno (Morán et al. 1996). Los suelos dominantes son del tipo luvisol, regosol y cambisol (Alfaro 2004).

Clima: húmedo templado isotermal, con precipitaciones anuales de 1800 a 2 000mm (García 1973). De las cinco áreas en comparación, ésta tiene mayor influencia de la costa, por su cercanía a ella.

Vegetación: bosque tropical subcaducifolio, bosque mesófilo de montaña, bosque de pino y encino y bosque de pino.

Trabajo de campo efectuado por el MZFC entre 2006 y 2008, sobre nueve estaciones de muestreo y otros sitios con recolectas muy ocasionales; se reunieron finalmente en cinco localidades, homogéneas en tipo de vegetación y en un mismo intervalo altitudinal, bien representadas por el esfuerzo de recolecta (Ávalos et al. en prep.; Luis et al. en prep.).

Área de Teocelo-Xalapa (TEX), Veracruz.

Ubicación: sobre los $19^{\circ} 21^{\prime}-19^{\circ} 27^{\prime}$ N-96 $55^{\circ}-97^{\circ} 00^{\prime} \mathrm{W}$.

Clima: templado húmedo con precipitación anual en torno a los $1500 \mathrm{~mm}$.

Vegetación: bosque mesófilo de montaña y bosque tropical subcaducifolio.

Trabajo de campo: desde 1975 hasta 1981 y datos de colecciones y literatura, con un registro de 246 especies. Se toman siete estaciones de muestreo. Para Xalapa sólo se tomaron los datos procedentes del trabajo de campo en el Parque Francisco Javier Clavijero, pues muchos registros antiguos no pueden asimilarse con seguridad al área.

Sierra de Juárez (JUA), Oaxaca.

Ubicación: entre los $17^{\circ} 29^{\prime}-17^{\circ} 54^{\prime}$ N- $-96^{\circ} 19^{\prime}-97^{\circ} 44^{\prime} \mathrm{W}$.

Topografía: muy accidentada, alcanza los $3100 \mathrm{~m}$ de altitud.

Suelos: acrisol, cambisol, rendzina y feozem.

Clima: hasta la altitud de $1000 \mathrm{~m}$ el clima dominante es de tipo A (cálido), con los subtipos Af(m) con lluvias abundantes durante todo el año y Am. A mayor altitud predomina el clima templado $(\mathrm{C})$, con tres subtipos: $\mathrm{C}(\mathrm{fm})$ templado húmedo con abundantes lluvias en verano, $\mathrm{C}(\mathrm{m})$ y $\mathrm{Cb}$ templado húmedo con lluvias en verano.

Vegetación: selva alta perennifolia, bosque mesófilo de montaña y bosque de pino y encino.

Trabajo de campo: entre marzo de 1986 y mayo de 1988. Junto con los datos procedentes de la literatura previa se obtuvo un conjunto de 314 especies. Se recogen los datos procedentes de siete localidades. Se unieron como una misma localidad los datos de todas las estaciones de muestreo situadas en la cota de los $100 \mathrm{~m}$ de altitud y los de las dos estaciones de muestreo de $2800 \mathrm{msnm}$ 
CUADRO 2

Características de las localidades

TABLE 2

Traits of the different collection sites

\begin{tabular}{|c|c|c|c|c|c|c|c|c|}
\hline Localidad & Acr. & Ubicación & Sierra & Vert. & Altitud & Tipo de veg. & Esf. col. & N. esp. \\
\hline Agua Dulce & $\mathrm{AD}$ & $19^{\circ} 17^{\prime}-103^{\circ} 55^{\prime}$ & MAN & PAC & 250 & BTS & 39 & 129 \\
\hline Platanarillos & PT & $19^{\circ} 24^{\prime}-103^{\circ} 57^{\prime}$ & MAN & PAC & 350 & BTS & 45 & 135 \\
\hline La Calera & $\mathrm{LC}$ & $19^{\circ} 40^{\prime}-104^{\circ} 25^{\prime}$ & MAN & PAC & 600 & BTS & 53 & 155 \\
\hline Zenzontla & $\mathrm{ZE}$ & $19^{\circ} 39^{\prime}-104^{\circ} 04^{\prime}$ & MAN & PAC & 800 & BTC & 36 & 112 \\
\hline Ahuacapán & $\mathrm{AH}$ & $19^{\circ} 42^{\prime}-104^{\circ} 20^{\prime}$ & MAN & PAC & 900 & BTC & 36 & 128 \\
\hline Los Mazos & LM & $19^{\circ} 41^{\prime}-104^{\circ} 24^{\prime}$ & MAN & PAC & $1600-1750$ & BMM & 41 & 112 \\
\hline Rincón de Las Parotas & LP & $17^{\circ} 13^{\prime}-100^{\circ} 29^{\prime}$ & ATO & PAC & 300 & BTSCa & 32 & 123 \\
\hline Río Santiago & $\mathrm{RS}$ & $17^{\circ} 15^{\prime}-100^{\circ} 19^{\prime}$ & ATO & PAC & 680 & BTSCa & 78 & 140 \\
\hline Puente de Los Lugardo & PL & $17^{\circ} 20^{\prime}-100^{\circ} 15^{\prime}$ & ATO & PAC & $800-900$ & BTSCa & 24 & 125 \\
\hline El Faisanal & $\mathrm{EF}$ & $17^{\circ} 27^{\prime}-100^{\circ} 10^{\prime}$ & ATO & PAC & 1250 & BTSBMM & 40 & 134 \\
\hline Nueva Delhi & ND & $17^{\circ} 25^{\prime}-100^{\circ} 12^{\prime}$ & ATO & PAC & $1350-1450$ & $\mathrm{BMMCa}$ & 24 & 111 \\
\hline Los Retrocesos & LR & $17^{\circ} 25^{\prime}-100^{\circ} 11^{\prime}$ & ATO & PAC & $1580-1650$ & BMMCa & 16 & 96 \\
\hline La Golondrina & LG & $17^{\circ} 12^{\prime}-100^{\circ} 26^{\prime}$ & ATO & PAC & 1800 & BMM & 22 & 66 \\
\hline Descanso-Iris & DI & $17^{\circ} 28^{\prime}-100^{\circ} 11^{\prime}$ & ATO & PAC & $1950-2150$ & $\operatorname{BEBMM}(\mathrm{BL})$ & 22 & 49 \\
\hline Puerto del Gallo & PG & $17^{\circ} 28^{\prime}-100^{\circ} 10^{\prime}$ & ATO & PAC & $2450-2500$ & ВРЕВMМ & 24 & 61 \\
\hline El Azulillo & $\mathrm{AZ}$ & $15^{\circ} 53^{\prime}-96^{\circ} 29^{\prime}$ & LOX & PAC & 380 & BTS & 60 & 139 \\
\hline Pluma Hidalgo & $\mathrm{PH}$ & unión de datos & LOX & PAC & $1170-1190$ & BMM & 92 & 126 \\
\hline La Pasionaria & PS & unión de datos & LOX & PAC & $1440-1650$ & BMM & 100 & 129 \\
\hline San José del Pacífico & SJ & unión de datos & LOX & PAC & $2050-2400$ & $\mathrm{BPE}$ & 52 & 68 \\
\hline Doncella-Nevería & $\mathrm{DN}$ & unión de datos & LOX & PAC & $2660-2820$ & $\mathrm{BP}$ & 32 & 20 \\
\hline Barranca de Coyoapa & $\mathrm{BC}$ & $19^{\circ} 12^{\prime}-96^{\circ} 16^{\prime}$ & TXA & ATL & $600-900$ & BTSBE & - & 183 \\
\hline Puente de Texolo & $\mathrm{TX}$ & $19^{\circ} 24^{\prime}-97^{\circ} 00^{\prime}$ & TXA & ATL & 1100 & BMMBTS & - & 113 \\
\hline El Trapiche & ET & $19^{\circ} 21^{\prime}-96^{\circ} 58^{\prime}$ & TXA & ATL & 1150 & $\mathrm{BMMCa}$ & - & 132 \\
\hline Santa Rosa & SR & $19^{\circ} 23^{\prime}-96^{\circ} 59^{\prime}$ & TXA & ATL & 1220 & BMM & - & 143 \\
\hline Teocelo & $\mathrm{TE}$ & $19^{\circ} 23^{\prime}-96^{\circ} 58^{\prime}$ & TXA & ATL & 1220 & $\mathrm{BMMCa}$ & - & 117 \\
\hline Xalapa & XA & $19^{\circ} 32^{\prime}-96^{\circ} 55^{\prime}$ & TXA & ATL & 1350 & BMM & - & 136 \\
\hline Coatepec & $\mathrm{CO}$ & $19^{\circ} 27^{\prime}-96^{\circ} 57^{\prime}$ & TXA & ATL & 1500 & $\mathrm{BMMCa}$ & - & 150 \\
\hline Juárez-100 & $\mathrm{JB}$ & unión de datos & JUA & ATL & 100 & ВTP & 160 & 242 \\
\hline Cerro Armadillo & $\mathrm{CA}$ & $17^{\circ} 00^{\prime}-97^{\circ} 00^{\prime}$ & JUA & ATL & 250 & ВTP & 29 & 123 \\
\hline Soyolapan El Bajo & $\mathrm{SB}$ & $17^{\circ} 40^{\prime}-97^{\circ} 28^{\prime}$ & JUA & ATL & 300 & BTP & 15 & 161 \\
\hline Puerto Eligio & $\mathrm{PE}$ & $17^{\circ} 40^{\prime}-97^{\circ} 28^{\prime}$ & JUA & ATL & 650 & ВТРВММ & 31 & 174 \\
\hline Metates & $\mathrm{ME}$ & $17^{\circ} 35^{\prime}-97^{\circ} 28^{\prime}$ & JUA & ATL & 900 & ВММВТР & 60 & 228 \\
\hline La Esperanza & $\mathrm{LE}$ & $17^{\circ} 37^{\prime}-97^{\circ} 34^{\prime}$ & JUA & ATL & 1750 & BMM & 81 & 154 \\
\hline Juárez-Alto & JA & unión de datos & JUA & ATL & 2800 & BPE & 8 & 33 \\
\hline
\end{tabular}

Acr.=Acrónimo; Vert.=Vertiente; Altitud=Altitud, en Msnm; Tipo de veg.=Tipo de Vegetación de acuerdo con Rzedowski (1978); Esf. col.=Esfuerzo de colecta, en personas-día; N.esp.=Número de especies, a efectos de este estudio; ATO=sierra de Atoyac de Álvarez; MAN=sierra de Manantlán; JUA=sierra de Juárez; TXA=área montañosa de Teocelo - Xalapa; $\mathrm{PAC}=$ Vertiente del Pacífico; ATL=Vertiente del Atlántico; BTS=Bosque tropical subcaducifolio; Ca=Perturbación por establecimiento de cafetales; $\mathrm{BMM}=$ bosque mesófilo de montaña; $\mathrm{BE}=\mathrm{B}$ osque de encino; $\mathrm{BL}=\mathrm{Bosque}$ de lauráceas (tipo particular de $\mathrm{BMM}$ ); $\mathrm{BPE}=$ Bosque de pino y encino; $\mathrm{BTC}=$ Bosque tropical caducifolio; $\mathrm{BTP}=$ Bosque tropical perennifolio. 
Papilionidae, Pieridae y Nymphalidae, por el buen conocimiento taxonómico y biogeográfico que se tiene de ellas (Llorente et al. 1997, Luis et al. 2003, Vargas et al. 2008, Luis et al. 2010). A fin de normalizar o unificar la nomenclatura, las listas procedentes de los trabajos originales se actualizaron con la lista taxonómica más reciente para México (Llorente et al. 2006). Se decidió trabajar a nivel subespecífico, considerado más informativo para definir las relaciones entre las áreas (Luna 1994), debido a que en algunos casos, en cada sierra se encuentra una subespecie característica, aunque ésta también suele ser la misma en sierras adyacentes o pertenecientes a la misma unidad orográfica.

Con los registros procedentes de todas las estaciones de muestreo, se construyó la matriz de presencia (1)-ausencia (0); esta matriz (398 táxones x 34 localidades) muestra los datos de distribución de los táxones. La configuración de esta matriz se realizó con el soporte del programa Excel, de Office 2010 para Windows.

Para examinar la riqueza $v s$. altitud en las cinco unidades orográficas estudiadas, se representó el comportamiento y las líneas de tendencia para las cinco unidades mediante el uso de Excel. Además se calculó una regresión lineal entre el logaritmo de la riqueza y la altitud para el total de localidades.

Se calculó la similitud faunística para todas las localidades de las cinco sierras, mediante el índice de similitud de Sørensen $[\mathrm{QS}=2 \mathrm{~s} /(\mathrm{a}+\mathrm{b})$; donde $\mathrm{s}$ corresponde al número de especies compartidas por cada par de inventarios, mientras que a y b son el número total de especies en cada uno de ellos]. Este coeficiente es uno de los de uso más generalizado en este tipo de estudios (Pielou 1975, Magurran 1988). El valor de este índice aplicado a pares de comunidades de igual peso equivale a una medida directa de la similitud o solapamiento, matemáticamente muy robusta, derivada de la diversidad beta verdadera con datos de presencia-ausencia (Jost 2006, Jost 2007).

A partir de la matriz de presencia-ausencia se obtuvo la matriz de similitud con la opción DICE para similitud faunística del programa informático NTSYSpc 2.02k (Applied Biostatistics Inc.) (Rohlf 1988), a partir de la cual se generó el fenograma general de áreas, mediante la técnica del agrupamiento promedio no ponderado (UPGMA).

\section{RESULTADOS}

Distribución altitudinal de la riqueza: Las figuras 2 (vertiente pacífica) y 3 (vertiente atlántica) muestran la variación de la riqueza específica (eje de ordenadas) con la altitud (eje de abscisas) para todas las estaciones y las líneas de tendencia para las cinco unidades montañosas. Se observa una tendencia general a la disminución de la riqueza con el incremento de altitud.

En las sierras de Manantlán, Atoyac de Álvarez y Loxicha, se presenta una tendencia más definida en la disminución de la riqueza al incrementar la altitud, aunque en las dos primeras se observa un pequeño incremento de diversidad a altitudes intermedias. En la sierra de Juárez este patrón no está bien definido, mientras que en todas las localidades del área montañosa de Teocelo-Xalapa (600-1 500m) se observa un número similar de especies.

Fenograma general de áreas: La figura 4 muestra el fenograma general de áreas, donde se definen tres grupos; el primero se separa principalmente por efecto de la altitud y se detalla con un cuadro en la parte inferior. Los siguientes grupos están separados por su ocurrencia en cada una de las vertientes estudiadas: se ubica en la parte superior los correspondientes a la vertiente pacífica (enmarcado); el segundo grupo se refiere a los sitios de la vertiente atlántica. De esta forma se caracterizan los siguientes grupos:

1. Elevaciones superiores (JA-DN): Se distinguen dos grandes grupos: el primero (en la presentación de la figura 4, compuesto por los inventarios de JA y DN), muy alejado del resto, se caracteriza porque sus componentes presentan un bajo grado de similitud entre ellos. Son los dos únicos inventarios 


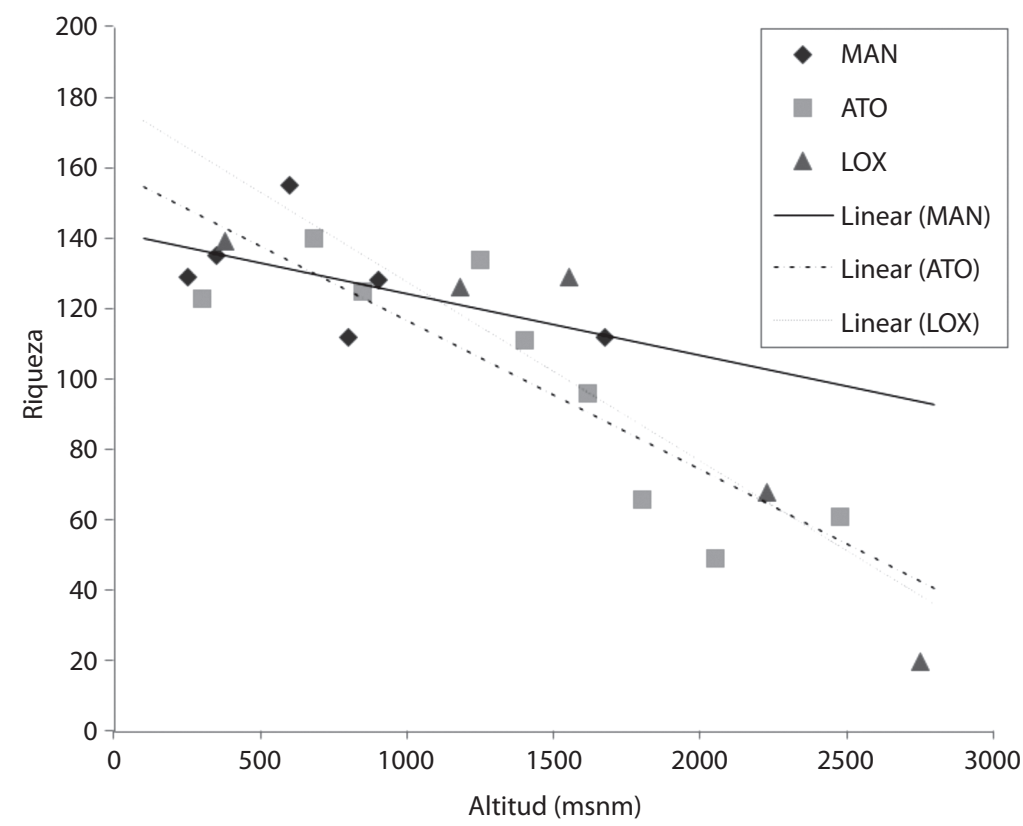

Fig. 2. Relación de la riqueza con la altitud para las zonas evaluadas en la vertiente pacífica de México. Fig. 2. Relationship between richness with altitude for the evaluated areas on the Pacific side of Mexico.

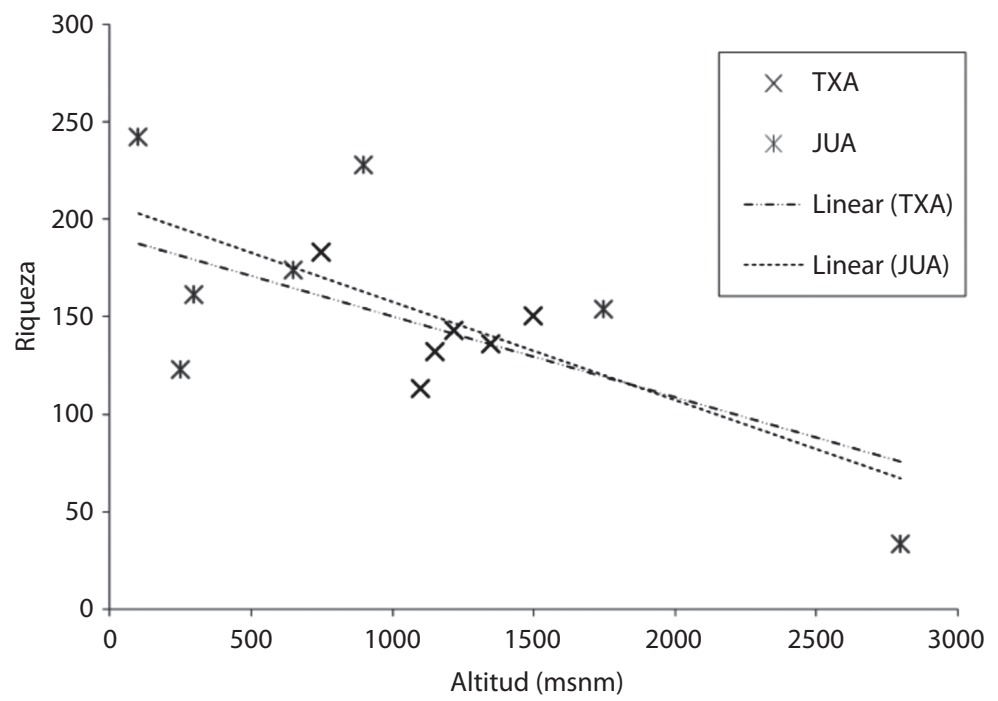

Fig. 3. Relación de la riqueza con la altitud para las zonas evaluadas en la vertiente atlántica de México. Fig. 3. Relationship between richness with altitude for the evaluated areas on the Atlantic side of Mexico.

procedentes de elevaciones superiores a los $2500 \mathrm{msnm}$, caracterizados por faunas pobres -riqueza específica inferior a $35 \mathrm{y}$ ausencia de Papilionidae- más que por la presencia de elementos compartidos entre ellos. No se identificaron especies exclusivas de este estrato altitudinal, aunque sí algunas típicas de altitudes elevadas como Hesperocharis graphites H.W. Bates, 1864, Phyciodes mylitta (W.H. Edwards, 


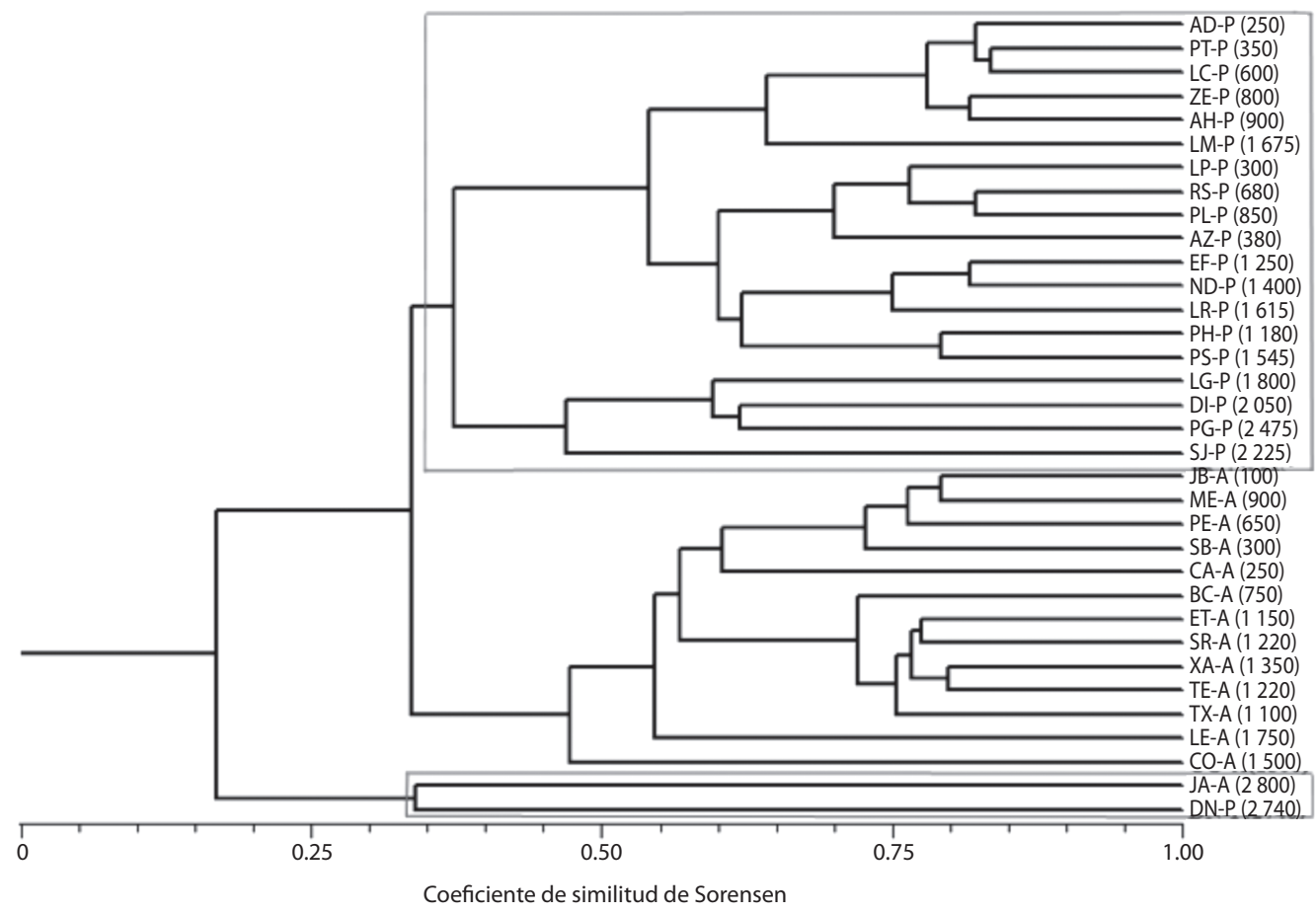

Fig. 4. Fenograma de similitud general. Fig. 4. General similarity cluster.

Tras el acrónimo de la localidad se indica la vertiente a la cual pertenece: P (Pacífico), A (Atlántico); y entre paréntesis la altitud, en metros sobre el nivel del mar.

1861) o Anetia thirza Geyer, [1833] y, en algunos casos (Catasticta teutila Doubleday, 1847 o Paramacera xicaque Reakirt, [1867]), hay diferenciación subespecífica en las dos vertientes.

2. Elevaciones baja, medias y altas (AD-CO): El segundo grupo muestra las relaciones de mayor similitud y comprende las 32 estaciones restantes (en la figura 4, AD a CO). Se subdivide $(\mathrm{QS}=0.33)$ en dos grandes bloques: el primero (AD-SJ) compuesto por los inventarios de la vertiente pacífica; y el segundo (JB-CO) conformado por las localidades atlánticas.

Así, aunque hay táxones de amplia distribución representados en todas las sierras (v.g. Heraclidas thoas autocles (Rothschild \& Jordan, 1906), Chlosyne lacinia (Geyer, 1837), Smyrna blomfildia datis Fruhstorfer, 1908 o Biblis hyperia
Boisduval, 1836), muchos son exclusivos de una u otra vertiente. En algunos casos existen distintas especies para un mismo género como Battus eracon (Godman \& Salvin, 1837) en la vertiente pacífica y B. ingenuus (Dyar, 1907) o B. lycidas (Cramer, 1777) en la vertiente atlántica. Mientras que en otros hay diferenciación subespecífica (Battus laodamas iopas (Godman \& Salvin, 1897) y Enantia mazai diazi Llorente, 1984 en la vertiente pacífica frente a B. l. copanae Reakirt, 1873 y $E$. m. mazai Llorente, 1984 en la vertiente del Golfo. También podemos encontrar táxones menos relacionados restringidos a una de las dos vertientes: Catonephele cortesi R.G. Maza, 1982, común a los inventarios de la vertiente atlántica; el género Ithomia Hübner, 1816 o Dryadula phaetusa (Linnaeus, 1758) en la vertiente atlántica. 
2.1. Vertiente pacífica (AD-SJ): Dentro del bloque pacífico, las estaciones pertenecientes a la Sierra de Manantlán conforman un grupo compacto. Algunas especies se identifican ampliamente en esta sierra, como Anthanassa sitalces (Godman \& Salvin, 1882), mientras que son poco reconocidas en las otras unidades estudiadas; Consul electra adustus Lamas, 2003 es una subespecie característica de Manantlán, sustituida por C. e. electra (Westwood, 1850) en las otras sierras. También es exclusiva Pedalioides sp. nov.

Las localidades de las sierras de Atoyac de Álvarez y Loxicha se agrupan de acuerdo con el intervalo altitudinal: de altitud inferior a 1100 msnm (LP-PS) y de altitudes intermedias (1 100-1 800msnm) (LG-SJ). Finalmente, las localidades de Sierra Madre del Sur entre los 1 800msnm y los $2500 \mathrm{msnm}$ (LG-SJ) constituyen un conjunto (altas elevaciones), donde los correspondientes a la Sierra de Atoyac de Álvarez (LG, DI, $\mathrm{PG})$ forman un grupo relacionado con SJ, de la región Loxicha.

Algunos táxones que contribuyen a conformar las agrupaciones entre las altitudes inferiores de las regiones de Atoyac de Álvarez y los Loxicha son Parides erithalion trichopus (Rothschild \& Jordan, 1906) y Nica flavilla bachiana (R.G. Maza \& J. Maza, 1985). El empobrecimiento paulatino de las lepidóptero-faunas define la agrupación entre las altitudes mayores, donde pueden identificar táxones exclusivos compartidos, como Paramacera xicaque rubrosuffusa L.D. Miller, 1972 o Cyllopsis clinas (Godman \& Salvin, 1889).

2.2. Vertiente del Golfo de México (JB-CO): Se define un grupo conformado por las estaciones de altitudes entre 600 y $1350 \mathrm{msnm}$, que se divide en dos subgrupos muy compactos, según las unidades orográficas: Teocelo-Xalapa (BC-TX) y Sierra de Juárez (JB-CA); finalmente, se anexan las localidades LE (1 750msnm), de características intermedias entre el grupo de altitudes intermedias y el de elevaciones superiores, pero con una importante riqueza y CO (1500msnm), de más difícil interpretación.

Algunos táxones característicos de la lepidóptero-fauna del Golfo de México que sólo se identificaron en la lepidóptero-fauna de TeoceloXalapa fueron Microta elva horni Rebel, 1906, Diaethria astala astala (Guérin-Méneville, [1844]) o Limentis asthemis arizonensis W.H. Edwards, 1882, mientras que Opsiphanes quiteria quirinus Godman \& Salvin, 1881, Caligo uranus Herrich-Schäffer, 1850 o Itaballia pandosia kicaha (Reakirt, 1863) son exclusivos de la Sierra de Juárez.

\section{DISCUSIÓN}

Existe una clara tendencia a la disminución del número de especies de mariposas conforme se incrementa la altitud. Esta relación se manifiesta, sobre todo, en el drástico descenso de la diversidad a partir de los $1800 \mathrm{~m}$. En la literatura, se han descrito tres pisos altitudinales (de 600 a $1200 \mathrm{~m}$, de 1200 a $1800 \mathrm{~m}$ y por arriba de $1800 \mathrm{~m}$ ) de la fauna montana de acuerdo a su composición faunística, la cual es producto de los procesos históricos (Halffter 1976, Halffter 1987, Llorente 1984), en donde caracterizan estas faunas en función a su afinidad biogeográfica. La mayor riqueza en este trabajo, se presenta de los 600 a $1200 \mathrm{~m}$ de altitud, lo que concuerda con los datos de la literatura (Janzen 1973, Janzen et al. 1976, Haber 1978), que ecológicamente atribuyen esta diversidad a una mayor productividad. En transectos pequeños (v. gr. Teocelo-Xalapa) esta tendencia es difícil de visualizar, pues predomina la influencia de 
otros factores o particularidades de las propias localidades, también se puede observar, que en zonas montañosas aisladas los pisos altitudinales tienden a compactarse $v$. gr. Los Tuxtlas, Veracruz.

En este aspecto, cabe tener en cuenta que la distribución altitudinal de la fauna, se debe en realidad a muchas condiciones asociadas con la altitud (históricas-ecológicas), como la afinidad biogeográfica y diversificación de un taxon; así como de las condiciones de la temperatura, humedad o tipo de vegetación, entre otras, frente a las cuales cada especie reacciona de modo específico, de acuerdo con su valencia ecológica y la existencia de factores limitantes que condicionan su distribución (Udvardy 1969).

Con respecto al análisis de agrupamientos, este trabajo, con base en un gran número de inventarios implica comprender la distribución de la lepidóptero-fauna, con base en la influencia de diversos factores (altitud -intervalo, diferencia entre estaciones-, tipo de vegetación, unidad fisiográfica, intervalo de latitudes, aislamiento de las sierras, características del muestreo, y la pertenencia a una vertiente -Atlántica vs. Pacífica-) en la definición de éstos, por lo que la interpretación de los resultados resulta más compleja. Un fenograma con más de 30 sitios es difícil de interpretar, y más inventarios representan un problema casi imposible de descifrar de acuerdo con las ideas de Magurran (1988). Así, la definición de un piso intermedio es más clara al restringir el estudio a un número menor de regiones (Monteagudo et al. 2001). El fenograma presentado, al manejar 34 localidades, presenta estos problemas.

En este estudio, el factor altitudinal fue el más importante en la diferenciación de las lepidóptero-faunas, al definir dos grupos o separar rápidamente a las localidades superiores a los $2500 \mathrm{~m}$ de altitud y las inferiores; le sigue en importancia, su pertenencia a la vertiente (atlántica o pacífica), lo que sugiere que las faunas de mariposas están más definidas por factores biogeográficos que ecológicos. En la vertiente pacífica las estaciones se agruparon con base en dos factores: la pertenencia a una unidad geográfica (sierra) y el factor altitudinal (estaciones de elevaciones bajas -hasta los $1200 \mathrm{msnm}-$, intermedias -1200 a $1800 \mathrm{msnm}-\mathrm{y}$ altas -1800 a $2500 \mathrm{msnm}-$ ). En la vertiente atlántica las estaciones se agruparon de acuerdo a la unidad a la cual pertenecen, con una mayor diferenciación de los inventarios de más de $1500 \mathrm{~m}$ de altitud, aunque la distribución de las estaciones de muestreo puede influir en esta menor definición de los pisos. La distribución de la humedad en ambas vertientes también es fundamental para explicar la formación de los pisos altitudinales. En la vertiente atlántica se presenta una mayor precipitación y ésta es mucho más regular para todo el transecto; a diferencia de la pacífica, en donde la precipitación es más irregular en cuanto a cantidad y distribución, lo que se refleja en la asociación de la vegetación y la lepidóptero-fauna en pisos más definidos (Luis \& Llorente 1990).

La presencia de la fauna de mariposas en pisos altitudinales también es producto de su historia biogeográfica. De forma general, se observa que las especies con afinidad neártica están asociadas al tercer piso (Pterourus $m$. multicaudata (W.F. Kirby, 1884) y Nymphalis a. antiopa (Linnaeus, 1758)) y las de afinidad neotropical al primer piso (Oleria zea zea (Hewitson, [1855]), Mechanitis manapis doryssus H.W. Bates, 1864 у M. polymnia lycidice H.W. Bates, 1864). Para el piso intermedio, se tiene una serie de géneros que han evolucionado en la región mesoamericana y que se han diferenciado en cada una de las cadenas montañosas que se presentan en el país, entre los 1400 y los $1800 \mathrm{msnm}$ (Pereute Herrich-Schäffer, 1867, Enantia Hübner, [1819], Lieinix Gray, 1832, Memphis Hübner, 1819). Además, en este piso influye la migración altitudinal, en donde se observan táxones que suben del primer piso o bajan del tercero, lo que implica que su composición es más difícil de interpretar y que en algunas ocasiones no llega a identificarse.

Es necesario ampliar el estudio a más áreas montañosas, que sean representativas de un mayor intervalo latitudinal y que representen a cada una de las islas montañosas descritas 
en la literatura $v . g r$. Llorente 1984, para determinar la influencia de los distintos factores como la latitud y la pertenencia a una vertiente determinada; ello permitiría caracterizar la lepidóptero-fauna de distintas regiones con respecto a los mismos y así establecer un mayor conocimiento acerca de los patrones espaciales de diversidad de la biota mexicana.

\section{AGRADECIMIENTOS}

A nuestros compañeros del MZFC, Jorge Llorente Bousquets y José Luis Salinas Gutiérrez, quienes amablemente se prestaron a la revisión del manuscrito y contribuyeron a mejorarlo con su visión y acertadas propuestas. A los proyectos CONACyT 083237 y PAPIIT IN214212. A los revisores anónimos del manuscrito que enriquecieron este trabajo.

\section{RESUMEN}

Las mariposas diurnas integran uno de los grupos más utilizados para el reconocimiento y monitoreo de la diversidad de una biota. Se realizó un estudio comparativo de las faunas de mariposas de las familias Papilionidae, Pieridae y Nymphalidae de cinco áreas montañosas de México, situadas en las vertientes Atlántico y Pacífico: las sierras de Atoyac de Álvarez, Manantlán, Juárez, y las áreas de Teocelo-Xalapa y la región Loxicha, con un total de 34 localidades representativas de los cinco transectos altitudinales, que comprenden de los 300 a los $3100 \mathrm{~m}$ de altitud. Se observó una tendencia general a la disminución de la riqueza con la altitud. Se analizó la similitud entre el total de localidades mediante el índice de Sørensen, diferenciándose en primer lugar las dos estaciones de elevaciones superiores (sobre los $2500 \mathrm{msnm}$ ), caracterizadas por pobres lepidóptero-faunas. En el grupo principal (32 sitios) las principales agrupaciones se dan de acuerdo, en primer lugar, a la pertenencia a la vertiente pacífica o atlántica, evento más histórico que ecológico y, después, las estaciones de una misma sierra o según tres diferentes pisos altitudinales, que se caracterizan de acuerdo con los táxones predominantes.

Palabras clave: Papilionoidea, mariposas, distribución altitudinal, similitud faunística, índice de Sørensen.

\section{REFERENCIAS}

Alfaro, G. 2004. Suelos, p. 55-66. In A. García, M.J. Ordóñez \& M.A. Briones (eds.). Biodiversidad de
Oaxaca, Fondo oaxaqueño para la conservación de la naturaleza, WWF, Inst. Biología, UNAM, México, D.F., México.

Balcázar, M. 1993. Butterflies of Pedernales, Michoacán, México, with notes on seasonality and faunistic affinities (Lepidoptera: Papilionoidea and Hesperioidea). Trop. Lepid. 4: 93-105.

Beutelspacher, C.R. 1983. Mariposas diurnas de El Chorreadero, Chiapas (Insecta: Lepidoptera). An. Inst. Biol. Univ. Nac. Autón. Méx. 53: 341-366.

Comisión Nacional para el Conocimiento y Uso de la Biodiversidad (CONABIO). 2010. El Bosque Mesófilo de Montaña en México: Amenazas y Oportunidades para su Conservación y Manejo Sostenible. CONABIO, México, D.F., México.

García, E. 1973. Modificación del sistema de clasificación climática de Köppen. UNAM, México, D.F., México.

Haber, W.A. 1978. Evolutionary Ecology of Tropical Mimethic Butterflies (Lepidoptera: Ithominae). Ph.D. Thesis, University of Minnesota, Minnesota, EE.UU.

Halffter, G. 1976. Distribución de los insectos en la Zona de Transición Mexicana. Relaciones con la entomofauna de Norteamérica. Fol. Entomol. Mex. 35: 1-64.

Halffter, G. 1987. Biogeography of the montane entomofauna of Mexico and Central America. Ann. Rev. Entomol. 32: 95-114.

Hoffman, C.C. 1933. La fauna del Distrito del Soconusco (Chiapas). Un estudio zoogeográfico. An. Inst. Biol. Univ. Nac. Autón. Méx. 4: 211-242.

Janzen, D.H. 1973. Sweep samples of tropical foliage insects: description of study sites, with data on species abundance and size distribution. Ecology 54: 659-686.

Janzen, D.H., M. Ataroff, M. Fariñas, S. Reyes, N. Rincón, A. Soler, P. Soriano \& M. Vera. 1976. Changes in the arthropod community along an elevational transect in the Venezuelan Andes. Biotropica 8: 193-203.

Jost, L. 2006. Entropy and diversity. Oikos 113: 363-375.

Jost, L. 2007. Partitioning diversity into independent alpha and beta components. Ecology 88: 2427-2439.

Llorente, J. 1984. Sinopsis sistemática y biogeográfica de los Dismorphiinae de México con especial referencia al género Enantia Hüebner (Lepidoptera: Pieridae). Fol. Entomol. Mex. 58: 1-207.

Llorente, J., A. Garcés \& A. Luis. 1986. El paisaje teoceleño IV. Las mariposas de Jalapa-Teocelo, Veracruz. Teocelo 4: 14-37.

Llorente, J., A. Luis, I. Vargas \& J. Soberón. 1994. Biodiversidad de las mariposas: su conocimiento y su conservación en México. Rev. Soc. Mex. Hist. Nat. 14: 313-324.

Llorente, J., L. Oñate, A. Luis \& I. Vargas. 1997. Papilionoidae y Pieridae de México: distribución geográfica 
e ilustración. CONABIO, UNAM, S y G., México, D.F., México.

Llorente, J., A. Luis \& I. Vargas. 2006. Apéndice general de Papilionoidea: lista sistemática, distribución estatal y provincias biogeográficas, p. 945-1009. In J.J. Morrone \& J. Llorente (eds.). Componentes bióticos principales de la entomofauna mexicana. UNAM, México, D.F., México.

Luis, A. \& J. Llorente. 1990. Mariposas en el Valle de México: Introducción e Historia. 1. Distribución local y estacional de los Papilionoidea de la Cañada de los Dínamos, Magdalena Contreras, D.F., México. Fol. Entomol. Mex. 78: 95-198.

Luis, A., I. Vargas \& J. Llorente. 1991. Lepidopterofauna de Oaxaca I: distribución y fenología de los Papilionoidea de la sierra de Juárez. Publ. Espec. Mus. Zool. Fac. Ciencias, UNAM 3: 1-119.

Luis, A., J. Llorente \& I. Vargas. 2003. Nymphalidae de México I (Danainae, Apaturinae, Biblidinae y Heliconinae): distribución geográfica e ilustración. UNAM, CONABIO, México, D.F., México.

Luis, A., J. Llorente \& I. Vargas. 2005. Una megabase de datos de mariposas y la regionalización biogeográfica de México, p. 269-294. In J. Llorente \& J.J. Morrone (eds.). Regionalización biogeográfica en Iberoamérica y tópicos afines. CYTED-UNAM-CONABIO, México, D.F., México.

Luis, A., J. Llorente, I. Vargas \& C. Pozo. 2010. Nymphalidae de México III (Nymphalinae): distribución geográfica e ilustración. UNAM, El Colegio de la Frontera Sur, México, D.F., México.

Luna, I. 1994. Los conceptos de especie evolutiva y filogenética, p. 67-78. In J. Llorente \& I. Luna (eds.). Taxonomía Biológica. Serie Ediciones Universitarias, FCE, México, D.F., México.

Luna, I., L. Almeida \& J. Llorente. 1989. Florística y aspectos fitogeográficos del bosque mesófilo de montaña de las cañadas de Ocuilán, estados de Morelos y México. An. Inst. Biol. Univ. Nac. Autón. Méx. AM, Serie Botánica 59: 63-87.

Luna, I. \& O. Alcántara. 2001. Análisis de simplicidad de endemismos (PAE) para establecer un modelo de vicarianza preliminar del bosque mesófilo de montaña mexicano, p. 273-277. In J. Llorente \& J.J. Morrone (eds.). Introducción a la biogeografía en Latinoamérica: teorías, conceptos, métodos y aplicaciones. UNAM, México, D.F., México.
Magurran, A.E. 1988. Ecological diversity and its measurement. Princeton University, Nueva Jersey, EE.UU.

Monteagudo, D., A. Luis, I. Vargas \& J. Llorente. 2001. Patrones altitudinales de diversidad de mariposas en la Sierra Madre del Sur (México) (Lepidoptera: Papilionoidea). SHILAP Rvta. Lepid. 29: 207-237.

Morán, D., J. Corona \& G. Tolson. 1996. Uplift and subduction erosion in southwestern Mexico since the Oligocene: Pluton geobarometry constraints. Earth Planet. Sci. Lett. 141: 51-65.

Murguía, M. \& F. Rojas. 2001. Biogeografía cuantitativa, p. 39-47. In J. Llorente \& J.J. Morrone (eds.). Introducción a la biogeografía en Latinoamérica: teorías, conceptos, métodos y aplicaciones. UNAM, México, D.F., México.

Pielou, E.C. 1975. The interpretation of ecological data. Wiley, Nueva York, EE.UU.

Pozo, C., A. Luis, S. Uc-Tescum, N. Salas \& A. MayaMartínez. 2003. Butterflies (Papilionoidea and Hesperoidea) of Calakmul, Campeche, México. SW Nat. 48: 505-525.

Rohlf, F.J. 1988. NTSYSpc. Numerical taxonomy and multivariate analysis system. Exeter, Nueva York, EE.UU.

Rzedowski, J. 1978. Vegetación de México. Limusa, México, D.F., México.

Soberón, J. \& J. Llorente. 1993. The use of species accumulation functions for the prediction of species richness. Conserv. Biol. 7: 480-488.

Udvardy, M. 1969. Dynamic Zoogeography. Van Nostrand Reinhold, Nueva York, EE.UU.

Vargas, I., J. Llorente \& A. Luis. 1992. Listado lepidopterofaunístico de la Sierra de Atoyac de Álvarez en el Estado de Guerrero: Notas acerca de su distribución local y estacional (Rhopalocera: Papilionoidea). Fol. Entomol. Mex. 86: 41-178.

Vargas, I.F., J. Llorente \& A.M. Luis. 1999. Distribución de los Papilionoidea (Lepidoptera: Rhopalocera) de la Sierra de Manantlán (250-1 650msnm) en los estados de Jalisco y Colima. Publ. Espec. Mus. Zool., Fac. Ciencias, UNAM 11: 1-153.

Vargas, I., J. Llorente, A. Luis \& C. Pozo. 2008. Nymphalidae de México II (Libytheinae, Ithomiinae, Morphinae y Charaxinae): distribución geográfica e ilustración. UNAM, CONABIO, México, D.F., México. 